DOI https://doi.org/10.30525/978-9934-26-038-4-61

\title{
DIAGNOSTIC SIGNIFICANCE OF TOLL-LIKE RECEPTORS 4 IN CRITICAL PATIENTS WITH INFECTIOUS COMPLICATIONS OF THE RESPIRATORY ORGANS
}

\author{
Dmytriiev D. V. \\ Doctor of Medicine, \\ Professor at the Anaesthesiology and Intensive Care Department \\ National Pirogov Memorial Medical University
}

Nazarchuk O. A.

Doctor of Medicine,

Professor of the Microbiology Department

National Pirogov Memorial Medical University

\section{Melnychenko M. V.}

Postgraduate Student at the Anaesthesiology and Intensive Care Department National Pirogov Memorial Medical University

\section{Levchenko B. I.}

Postgraduate Student at the Anaesthesiology and Intensive Care Department National Pirogov Memorial Medical University

\author{
Bagniyk N. A. \\ Postgraduate Student at the Microbiology Department \\ National Pirogov Memorial Medical University \\ Vinnytsya, Ukraine
}

Introduction. Ventilator-associated respiratory infection (VARI) is a common hospital complication in the intensive care unit (ICU) associated with medical care [1]. Among which there are known ventilator-associated tracheobronchitis and ventilator-associated pneumonia (VAP), occuring in approximately $20 \%$ of patients who are on mechanical ventilation for more than 48 hours and the mortality rate is about $30 \%$ [2, 3].

Methods and criteria for the diagnosis of VAP are still controversial [4]. Investigation of new attempts for its quick diagnostics are of great importance for timely individual correction etiotropic antibiotic treatment. In recent years, attempts have been made to identify biological markers of infection manifestation $[6,7]$. 
Toll-like receptors (TLRs) recognize pathogenic microorganisms and generate an immediate protective response by inducing the production of pro-inflammatory cytokines [8]. TLRs of type 4 (TLR-4), which are expressed in different types of immune system cells, are associated with different states of cellular activation, immune protection, homeostasis, and various diseases, specifically recognize bacterial lipopolysaccharide, leading to the synthesis of pro-inflammatory cytokines and chemokines $[9,10,11]$. Nowadays it is widely studied for diagnostic and therapeutic purposes in infectious complications.

Aim. To evaluate the dynamics of TLR-4 serum levels and local microbial colonization in patients with infectious respiratory complications and different treatment strategies.

Material and methods. The study involved 40 patients who underwent artificial lung ventilation (ALV) for 48 hours or more. Infectious airway complications were diagnosed as VAP by all criteria according to the US Centers for Disease Control and Prevention (CDC).

All patients received standard comprehensive treatment according to the treatment protocols of the underlying pathology. Respiratory support was performed with a ventilator «Hamillton-C2» in ASV mode.

Patients were divided into two groups of 20 people each with different approaches to the treatment of VAP. In the main observation group, inhalation of decamethoxine-based quaternary ammonium compound (QAC) antiseptic through a nebulizer from the first day after the appearance of clinical signs of VAP was used simultaneously with the empirically prescribed standard antibiotic therapy. Treatment of patients in the comparison group was performed in accordance with generally accepted recommendations with the use of systemic antibacterial and symptomatic therapy.

The effectiveness of treatment was assessed by clinical indicators of the disease, data from microbiological examination of tracheobronchial secretion and the level of TLR-4 in serum. Also we determined the degree of total microbial colonization of the respiratory tract by the number of microorganisms in $1 \mathrm{ml}$ of material, expressed in $\log \mathrm{CFU} / \mathrm{ml}$.

Results. As a result of microbiological examination, 40 samples of biological material obtained from patients with VAP in the first days after the onset of the disease there were isolated and identified by morphological, tinctorial, cultural and biochemical properties 49 strains of opportunistic pathogens.

Patients in the control group who received only standard systemic antibiotic therapy had higher rates of airway colonization during the first week of treatment. On the seventh day, the number of opportunistic pathogens identified in these patients with VAP reached $\log (6.49 \pm 0.35) \mathrm{CFU} / \mathrm{ml}$, which 
was four orders of magnitude higher than the content of microorganisms in the inflammatory focus in patients of the observation group ( $p<0,001)$.

A significant decrease in the number of microorganisms in the tracheobronchial secretion of patients in the comparison group was observed not earlier than after 10 days of receiving systemic antibiotic therapy. Thus, after 14 days it was found that the total number of microorganisms in $1 \mathrm{ml}$ of tracheobronchial aspirate expressed in log decreased almost twice compared to the seventh day, and was $(3.44 \pm 0.23) \mathrm{CFU} / \mathrm{ml}$, which significantly exceeded in 13 times this figure in observation group $(\mathrm{p}<0,001)$.

When using inhaled antiseptic drug based on decamethoxine in patients with VAP, a smaller increase in serum TLR-4 levels was found in the early period from the beginning of treatment. Thus, on the third day in patients of the observation group noted 1.42 times lower level of TLR-4 than in the comparison group $(\mathrm{p}<0,001)$.

In the comparison group, a significant increase in serum levels of TLR-4 ( $p<0.001$ ) was found at the same time with increased colonization by gramnegative bacteria in the tracheobronchial aspirate on the third day $(\mathrm{r}-\mathrm{Pirson}=$ 0.880).

The positive dynamics of the decrease the number of microorganisms in the aspirate obtained from the patients of the main group was marked by a decrease in the level of TLR-4 on the seventh day of treatment. Evidence of high efficacy of additional use of decamethoxine-based QAC in the complex treatment of patients with VAP is a strong correlation between a decrease in gram-negative pathogens of respiratory infections and a decrease in TLR-4 to almost baseline ( $\mathrm{r}$-Pirson $=0.893$ ). In comparison, the level of TLR-4 in the serum of patients with VAP who received only systemic therapy remained twice as high $(\mathrm{p}<0.001)$ than in the main group, and correlated with inflated levels of gram-negative microorganisms in the tracheobronchial aspirate of patients ( $\mathrm{r}$-Pirson $=0.801$ ).

\section{Conclusions.}

1. The level of TLR-4 in serum can serve as a criterion for the diagnosis of VAP and be a reliable marker of rational antibiotic therapy.

2. Early inhalation use of decamethoxine-based QAC in patients with severe infectious complications (VAP) is accompanied by significant changes in the qualitative composition of opportunistic respiratory microbiota.

3 . The positive dynamics of reducing the number of gram-negative microorganisms in the airway aspirate of patients with VAP by inhalation of decamethoxine-based antiseptic is marked by a slight increase in serum TLR-4 in the early period (3rd day) and closely correlates with 1,42 times faster reducing their level to baseline $(r-P i r s o n=0.893)$ on the seventh day of treatment. 
4. Determination of TLR-4 level can be used to assess the effectiveness of different approaches to the treatment of infectious complications of the respiratory system and to reflect the dynamics of the infectious process.

\section{References:}

1. Zarb P. The European Centre for Disease Prevention and Control (ECDC) pilot point prevalence survey of healthcare-associated infections and antimicrobial use. The European Centre for Disease Prevention and Control (ECDC) pilot point prevalence survey of health. Euro surveil 2012; 17:pii:20316. https://ecdc.europa.eu/sites/portal/files/media/en/publications/ Publications/healthcare-associated-infections-antimicrobial-use-PPS.pdf.

2. Martin-Loeches I, Povoa P, Rodríguez A, et al. Incidence and prognosis of ventilator-associated tracheobronchitis (TAVeM): a multicentre, prospective, observational study. Lancet Resp Med. 2015;3:859-68.

3. Chastre J, Fagon J-Y. Ventilator-associated pneumonia. Am J Respir Crit Care Med 2002;65:867-903

4. Chastre J, Fagon J-Y. Ventilator-associated pneumonia. Am J Respir Crit Care Med 2002;65:867-903

5. Fagon JY, Chastre J, Wolff M, et al. Invasive and noninvasive strategies for management of suspected ventilator-associated pneumonia. A randomized trial. Ann Intern Med 2000;132:621-3

6. Conway Morris A, Kefala K, Simpson AJ, et al. Evaluation of diagnostic methodology on the reported incidence of ventilator-associated pneumonia. Thorax 2009;64:516-22

7. Seligman R, Papassotiriou J, Morgenthaler N, et al. Copeptin, a novel prognostic biomarker in ventilator-associated pneumonia. Crit Care 2008; 12:R11.

8. O'Neill LA. TLRs: Professor Mechnikov, sit on your hat. Trends in immunology. 2004;25(12):687-93.

9. Liew FY, Xu D, Brint EK, O’Neill LA. Negative regulation of toll-like receptor-mediated immune responses. Nature reviews Immunology. 2005;5(6):446-58.

10. Janssens S, Beyaert $\mathrm{R}$. Role of toll-like receptors in pathogen recognition. Clin Microbiol Rev (2003) 16:637-4610.1128/CMR.16.4.637646.2003

11. Kanzler H, Barrat FJ, Hessel EM, Coffman RL. Therapeutic targeting of innate immunity with toll-like receptor agonists and antagonists. Nat Med (2007) 13:552-910.1038/nm1589 